\title{
Dual vibration resonance frequency CARS microscopy imaging of basal cell carcinoma to achieve stain free histopathology
}

Norbert Kiss ${ }^{1,2}$, Ádám Krolopp ${ }^{3}$, Kende Lőrincz ${ }^{1}$, Róbert Szipőcs ${ }^{2,3}$ and Norbert Wikonkál ${ }^{1}$

1 Department of Dermatology, Semmelweis University, Budapest, Hungary

2 Wigner RCP, Institute for Solid State Physics and Optics, Budapest, Hungary

3 R\&D Ultrafast Lasers Ltd, Budapest, Hungary

Basal cell carcinoma (BCC) is the most common malignancy in Caucasians and its incidence is increasing [1].

Its is of great importance to properly estimate the tumor borders before surgery as incomplete resection of the tumor will result in recurrence. Nonlinear microscopy has been previously utilized for the imaging of BCC in different experimental setups. To date, these experiments were carried out solely on frozen sections, and the captured images do not correlate with the standard hematoxylin and eosin (H\&E) staining [2]. Recently, Freudiger et al. introduced a novel method to visualize tissue morphology analogous to H\&E staining, using coherent anti-Stokes Raman scattering (CARS) technique [3].

In our present work, we introduce a novel algorithm to postprocess images obtained from dual vibration resonance frequency (DVRF) CARS measurements to acquire high-quality pseudo H\&E images of ex vivo BCC samples. We adapted our CARS setup to utilize the distinct vibrational properties of $\mathrm{CH}_{3}$ (mainly in proteins) and $\mathrm{CH}_{2}$ bonds (primarily in lipids). In a narrowband setup, the central wavelength of the pump laser is set to $791 \mathrm{~nm}$ and $796 \mathrm{~nm}$ to obtain optimal excitation. Due to the partial overlap of the excitation spectra and the $5-10 \mathrm{~nm}$ FWHM spectral bandwidth of our lasers, we set the wavelengths to $790 \mathrm{~nm}$ (for proteins) and $800 \mathrm{~nm}$ (for lipids). Nonresonant background from water molecules also reduces the chemical selectivity which can be significantly improved if we subtract the DVRF images from each other. As a result, we acquired two images: one for "lipids" and one for "proteins" when we properly set a multiplication factor to minimize the non-specific background. By merging these images, we obtained high contrast H\&E "stained" images of BBC's.

Nonlinear microscope systems upgraded for real time DVRF CARS measurements, providing pseudo H\&E images can be suitable for in vivo assessment of BCC in the future. With the utilization of such imaging, dermatosurgeons can plan the optimal safety margin before surgery to avoid incomplete removal of BCC's and the rate of recurrence can be minimized.

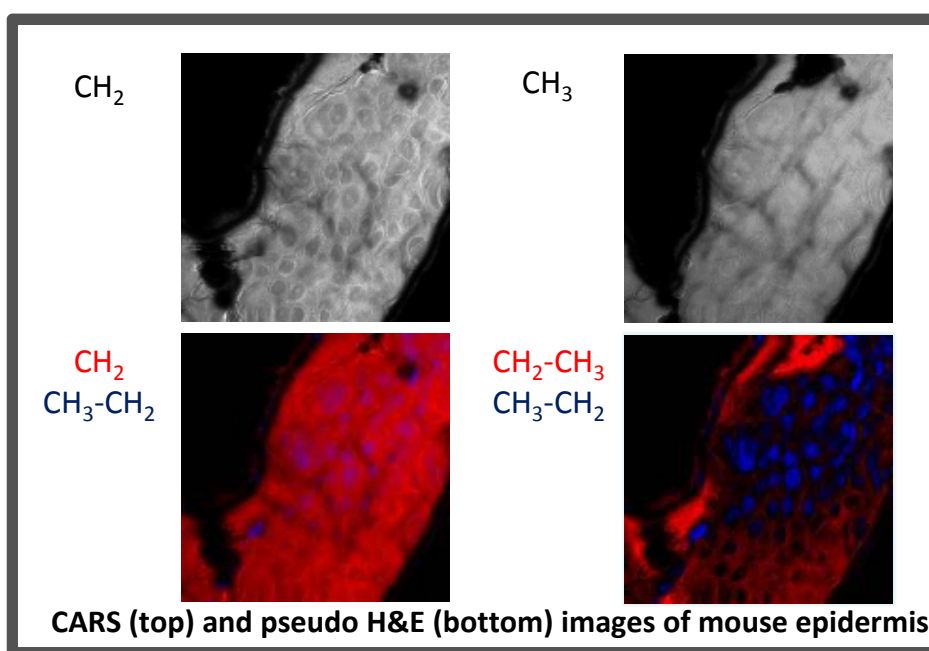

CARS (top) and pseudo H\&E (bottom) images of mouse epidermis

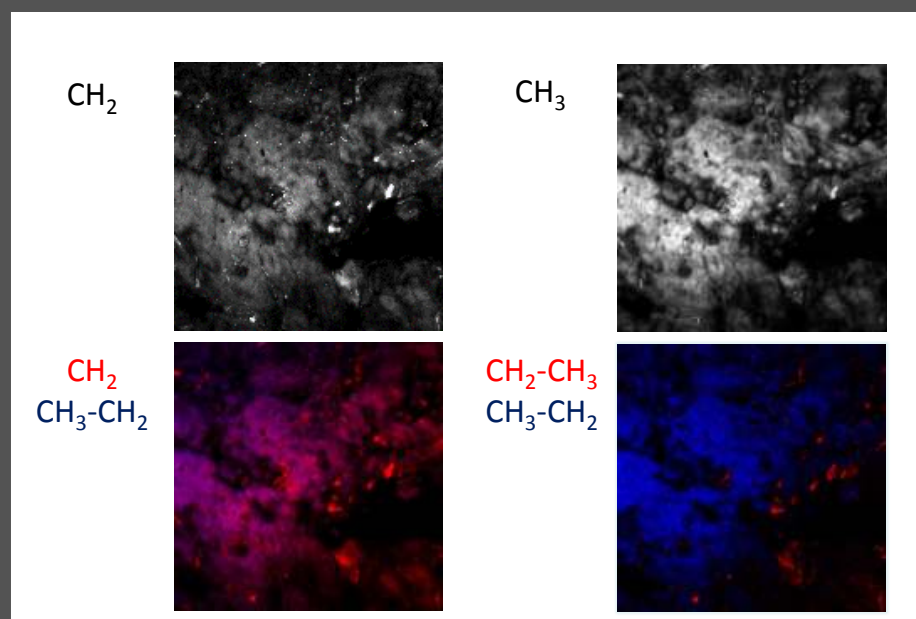

CARS (top) and pseudo H\&E (bottom) images of human dermis
$\mathrm{CH}_{2}$

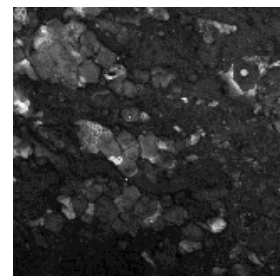

$\mathrm{CH}_{2}$

$\mathrm{CH}_{3}-\mathrm{CH}_{2}$

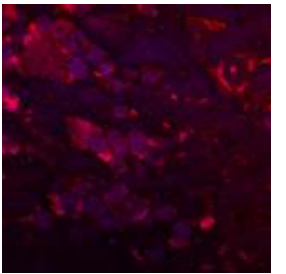

$\mathrm{CH}_{3}$

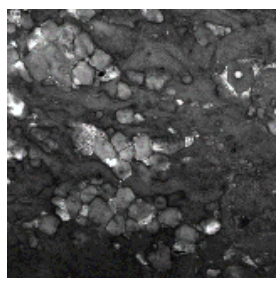

$\mathrm{CH}_{2}-\mathrm{CH}_{3}$ $\mathrm{CH}_{3}-\mathrm{CH}_{2}$

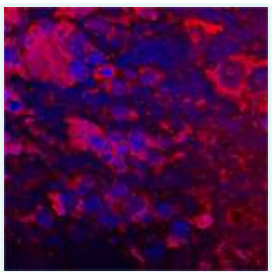

CARS (top) and pseudo H\&E (bottom) images of human BCC

\section{References}

1. Marzuka AG et al. (2015) Basal cell carcinoma: pathogenesis, epidemiology, clinical features, diagnosis, histopathology, and management. The Yale journal of biology and medicine $88(2): 167-179$

2. Seidenari S et al. (2013) Diagnosis of BCC by multiphoton laser tomography. Skin research and technology 19 (1):e297-304.

3. Freudiger CW, Pfannl R, Orringer DA, Saar BG, Ji M, Zeng Q, Ottoboni L, Wei Y, Waeber C, Sims JR, De Jager PL, Sagher O, Philbert MA, Xu X, Kesari S, Xie XS, Young GS (2012) Multicolored stain-free histopathology with coherent Raman imaging. Laboratory investigation 92 (10):1492-1502. 